\title{
Learning from the Kernel and the Range Space
}

\author{
Kar-Ann Toh \\ School of Electrical and Electronic Engineering \\ Yonsei University, Seoul, Korea \\ katoh@yonsei.ac.kr \\ IEEE/ACIS 17th ICIS 2018, pp.417-422 \\ Camera-ready finalized on 22 April 2018, paper presented on 07 June 2018 in ICIS \\ (Some typos have been fixed in current version)
}

\begin{abstract}
In this article, a novel approach to learning a complex function which can be written as the system of linear equations is introduced. This learning is grounded upon the observation that solving the system of linear equations by a manipulation in the kernel and the range space boils down to an estimation based on the least squares error approximation. The learning approach is applied to learn a deep feedforward network with full weight connections. The numerical experiments on network learning of synthetic and benchmark data not only show feasibility of the proposed learning approach but also provide insights into the mechanism of data representation.
\end{abstract}

\section{Introduction}

The learning problem in machine intelligence has been traditionally formulated as an optimization task where an error metric is minimized. In the system of linear equations, because it is difficult to have an exact match between the sample size and the number of model parameters, an approximation is often sought-after according to the primal solution space or the dual solution space in the least error sense. Such an optimization, particularly one that is based on minimizing the least squares error, has been a popular choice due to its simplicity and tractability in analysis and implementation. The approach is predominant in engineering applications as evident from its pervasive adoption in statistical and network learning.

Attributed to the computational effectiveness of the backpropagation algorithm running on the then limited hardware (see e.g., [1, 2, 3, 4, 5]) and the theoretical establishment of the mapping capability (see e.g., [6, 7, 8, 9]), the multilayer neural networks were once a popular tool for research and applications in the 1980s. With the advancement of computing facilities in the 1990s-2000s, such minimization of the error cost function had been progressed to the more memory intensive search algorithms utilizing the first- and the second-order methods of gradient descent (see e.g., [10, 11, 12]). Recently, driven by another leap bound advancement in the computing resources together with the availability of a large quantity of data, the multilayer neural networks reemerged as deep learning networks [13]. In view of the more demanding task of processing a large quantity of data with the highly 
complex network function on the limited computing resources such as the operating memory and the level of data vectorization, the backpropagation remained a viable tool for the optimization search.

In this work, we explore into utilization of the Kernel And the Range space (abbreviated as KAR space) for network learning. This approach exploits the approximation property of the kernel and the range space of the system of linear equations for learning the network weights. The main advantage of this approach is that neither descent nor gradient computation is needed for network learning. Moreover, once the network has been initialized, the learning can be calculated in a single operating pass where no iterative search is needed. The proposed approach can be applied to networks of arbitrary number of layers. This proposal opens up a new way of solving the network and functional learning problems without having to compute the gradient.

\section{The Kernel and the Range Space}

Consider the system of linear equations given by

$$
\mathbf{X} w=\mathbf{y}
$$

where $\mathbf{X} \in \mathbb{R}^{m \times d}$ is the data matrix, $\mathbf{y} \in \mathbb{R}^{m \times 1}$ is the target vector, and $\boldsymbol{w} \in \mathbb{R}^{d \times 1}$ is the unknown parameter vector to be solved. The range or image of a matrix is the span of its column vectors. The range of the corresponding matrix transformation is called the column space of the matrix. The kernel or the null space of a linear map is the set of solutions to the homogeneous equation $\mathbf{X} \boldsymbol{w}=\mathbf{0}$. In other words, $\boldsymbol{w}$ is in the kernel of $\mathbf{X}$ if and only if $\boldsymbol{w}$ is orthogonal to each of the row vectors of $\mathrm{X}$.

For an under-determined system (1) where $m<d$, the number of equations is less than the unknowns. This gives rise to an infinite number of solutions. However, a least norm solution can be obtained by constraining $\boldsymbol{w} \in \mathbb{R}^{d}$ to its $\mathbb{R}^{m}$ subspace [14] by utilizing the row space of $\mathbf{X}$, i.e., $\hat{\boldsymbol{w}}=\mathbf{X}^{T}\left(\mathbf{X X}^{T}\right)^{-1} \mathbf{y}$. Here, $\mathbf{X X}^{T}$ constitutes the kernel and is also known as the Gram matrix.

For an over-determined system where $m>d$, the $m$ equations in (11) are generally unsolvable when a strict equality is desired (see e.g., [19]). However, by multiplying $\mathbf{X}^{T}$ to both sides of (1), the resulted $d$ equations

$$
\mathbf{X}^{T} \mathbf{X} \boldsymbol{w}=\mathbf{X}^{T} \mathbf{y}
$$

is called the normal equation which can be rearranged to give the least squares error solution $\hat{\boldsymbol{w}}=\left(\mathbf{X}^{T} \mathbf{X}\right)^{-1} \mathbf{X}^{T} \mathbf{y}[19]$.

The above observations are summarized in the following results (see e.g., [16, 17, 18, 15]).

Lemma 1 Solving for $\boldsymbol{w}$ in the system of linear equations of the form (1) in the column space (range) of $\mathbf{X}$ or in the row space (kernel) of $\mathbf{X}$ is equivalent to solving the least squares error approximation problem. Moreover, the resultant solution $\hat{\boldsymbol{x}}$ is unique with a minimum-norm value in the sense that $\|\hat{\boldsymbol{w}}\|_{2}^{2} \leqslant\|\boldsymbol{w}\|_{2}^{2}$ for all feasible $\boldsymbol{w}$.

The proof is omitted here due to the space constraint. This result for systems with single output (output containing a single column, y) can be generalized to 
system with multiple outputs (output with multiple columns, $\mathbf{Y}=\left[\mathbf{y}_{1}, \cdots, \mathbf{y}_{q}\right]$ ) as follows.

Lemma 2 Solving for $\boldsymbol{W}$ in the system of linear equations of the form

$$
\mathbf{X} \boldsymbol{W}=\mathbf{Y}, \quad \mathbf{X} \in \mathbb{R}^{m \times d}, \boldsymbol{W} \in \mathbb{R}^{d \times q}, \mathbf{Y} \in \mathbb{R}^{m \times q}
$$

in the column space (range) of $\mathbf{X}$ or in the row space (kernel) of $\mathbf{X}$ is equivalent to minimizing the sum of squared errors given by

$$
\mathrm{SSE}=\operatorname{trace}\left((\mathbf{X} \boldsymbol{W}-\mathbf{Y})^{T}(\mathbf{X} \boldsymbol{W}-\mathbf{Y})\right) .
$$

Moreover, the resultant solution $\hat{\boldsymbol{W}}$ is unique with a minimum-norm value in the sense that $\|\hat{\boldsymbol{W}}\|_{2}^{2} \leqslant\|\boldsymbol{W}\|_{2}^{2}$ for all feasible $\boldsymbol{W}$.

Proof: Equation (3) can be re-written as a set of multiple linear systems of (1) as

$$
\mathbf{X}\left[\boldsymbol{w}_{1}, \cdots, \boldsymbol{w}_{q}\right]=\left[\mathbf{y}_{1}, \cdots, \mathbf{y}_{q}\right] .
$$

Since the trace of $(\mathbf{X} \boldsymbol{W}-\mathbf{Y})^{T}(\mathbf{X} \boldsymbol{W}-\mathbf{Y})$ is equal to the sum of the squared lengths of the error vectors $\mathbf{X} \boldsymbol{w}_{i}-\mathbf{y}_{i}, i=1,2, \ldots, q$, the unique solution $\hat{\boldsymbol{W}}=\left(\mathbf{X}^{T} \mathbf{X}\right)^{-1} \mathbf{X}^{T} \mathbf{Y}$ in the column space of $\mathbf{X}$ or that $\hat{\boldsymbol{W}}=\mathbf{X}^{T}\left(\mathbf{X X}^{T}\right)^{-1} \mathbf{Y}$ in the row space of $\mathbf{X}$, not only minimizes this sum, but also minimizes each term in the sum [20]. Moreover, since the column and the row spaces are independent, the sum of the individually minimized norms is also minimum.

The process of solving the algebraic equations under the kernel-and-range (KAR) spaces with implicit least squares error seeking shall be exploited to solve the network learning problem in the following section.

\section{$3 \quad$ Network Learning}

Consider an $n$-layer network (of $h_{1} \cdots h_{n}$ structure where $h_{n}=q$ is the output dimension) given by

$$
\mathbf{Y}=f_{n}\left(\left[\mathbf{1}, f_{n-1}\left(\cdots\left[\mathbf{1}, f_{2}\left(\left[\mathbf{1}, f_{1}\left(\mathbf{X} \boldsymbol{W}_{1}\right)\right] \boldsymbol{W}_{2}\right)\right] \cdots \boldsymbol{W}_{n-1}\right)\right] \boldsymbol{W}_{n}\right),
$$

where $\mathbf{X} \in \mathbb{R}^{m \times(d+1)}, \boldsymbol{W}_{1} \in \mathbb{R}^{(d+1) \times h_{1}}, \boldsymbol{W}_{2} \in \mathbb{R}^{\left(h_{1}+1\right) \times h_{2}}, \cdots, \boldsymbol{W}_{n-1} \in \mathbb{R}^{\left(h_{n-2}+1\right) \times h_{n-1}}$, $\boldsymbol{W}_{n} \in \mathbb{R}^{\left(h_{n-1}+1\right) \times q}, \mathbf{1}=[1, \ldots, 1]^{T} \in \mathbb{R}^{m \times 1}$, and $\mathbf{Y} \in \mathbb{R}^{m \times q}$. We shall partition the term $\boldsymbol{W}_{n}$ into $\left[\begin{array}{c}\mathbf{w}_{n}^{T} \\ \mathbf{W}_{n}\end{array}\right]$ where $\mathbf{w}_{n} \in \mathbb{R}^{q \times 1}$ and $\mathbf{W}_{n} \in \mathbb{R}^{h_{n-1} \times q}$. Assume that $f_{n}^{-1}$ exists, we can take the inverse of $f_{n}$ to both sides of (66). By separately considering the bias term and moving it to the left hand side, we can post-multiply both sides of the equation by $\mathrm{W}_{n}^{T}$ to get

$$
\begin{aligned}
& {\left[f_{n}^{-1}(\mathbf{Y})-\mathbf{1} \cdot \mathbf{w}_{n}^{T}\right] \mathbf{W}_{n}^{T}} \\
& \quad=f_{n-1}\left(\cdots\left[\mathbf{1}, f_{2}\left(\left[\mathbf{1}, f_{1}\left(\mathbf{X} \boldsymbol{W}_{1}\right)\right] \boldsymbol{W}_{2}\right)\right] \cdots \boldsymbol{W}_{n-1}\right) \cdot \mathbf{W}_{n} \mathbf{W}_{n}^{T}
\end{aligned}
$$


Next, by taking advantage of the column-row space manipulation, we arrive at

$$
\begin{aligned}
& \lim _{\lambda \rightarrow 0}\left[f_{n}^{-1}(\mathbf{Y})-\mathbf{1} \cdot \mathbf{w}_{n}^{T}\right] \mathbf{W}_{n}^{T}\left(\lambda \mathbf{I}+\mathbf{W}_{n} \mathbf{W}_{n}^{T}\right)^{-1} \\
& \quad=f_{n-1}\left(\cdots\left[\mathbf{1}, f_{2}\left(\left[\mathbf{1}, f_{1}\left(\mathbf{X} \boldsymbol{W}_{1}\right)\right] \boldsymbol{W}_{2}\right)\right] \cdots \boldsymbol{W}_{n-1}\right) \\
& {\left[f_{n}^{-1}(\mathbf{Y})-\mathbf{1} \cdot \mathbf{w}_{n}^{T}\right] \mathbf{W}_{n}^{T}\left(\mathbf{W}_{n} \mathbf{W}_{n}^{T}\right)^{-1}} \\
& \quad=f_{n-1}\left(\cdots\left[\mathbf{1}, f_{2}\left(\left[\mathbf{1}, f_{1}\left(\mathbf{X} \boldsymbol{W}_{1}\right)\right] \boldsymbol{W}_{2}\right)\right] \cdots \boldsymbol{W}_{n-1}\right) \\
& \quad\left[f_{n}^{-1}(\mathbf{Y})-\mathbf{1} \cdot \mathbf{w}_{n}^{T}\right] \mathbf{W}_{n}^{\dagger} \\
& \quad=f_{n-1}\left(\cdots\left[\mathbf{1}, f_{2}\left(\left[\mathbf{1}, f_{1}\left(\mathbf{X} \boldsymbol{W}_{1}\right)\right] \boldsymbol{W}_{2}\right)\right] \cdots \boldsymbol{W}_{n-1}\right),
\end{aligned}
$$

where $\dagger$ denotes the pseudo-inverse operation. This pseudo-inverse can be in the form of left or right operation depending on the matrix rank condition.

This process of inversion and moving the bias term plus kernel-and-range space manipulation is continued until reaching the first-layer where its hidden weights can be written as:

$$
\begin{aligned}
\boldsymbol{W}_{1}=\mathbf{X}^{\dagger} f_{1}^{-1}( & {\left[f _ { 2 } ^ { - 1 } \left(\cdots \left[f_{n-1}^{-1}\left(\left[f_{n}^{-1}(\mathbf{Y})-\mathbf{1} \cdot \mathbf{w}_{n}^{T}\right] \mathbf{W}_{n}^{\dagger}\right)\right.\right.\right.} \\
& \left.\left.\left.\left.-\mathbf{1} \cdot \mathbf{w}_{n-1}^{T}\right] \mathbf{W}_{n-1}^{\dagger} \cdots\right)-\mathbf{1} \cdot \mathbf{w}_{2}^{T}\right] \mathbf{W}_{2}^{\dagger}\right)
\end{aligned}
$$

After having $\boldsymbol{W}_{1}$ derived, it can be back-substituted to obtain $\boldsymbol{W}_{2}$ as

$$
\begin{aligned}
& \boldsymbol{W}_{2}=\left[\mathbf{1}, f_{1}\left(\mathbf{X} \boldsymbol{W}_{1}\right)\right]^{\dagger} f_{2}^{-1}\left(\left[f _ { 3 } ^ { - 1 } \left(\cdots \left[f _ { n - 1 } ^ { - 1 } \left(\left[f_{n}^{-1}(\mathbf{Y})\right.\right.\right.\right.\right.\right. \\
& \left.\left.\left.\left.\left.\left.-\mathbf{1} \cdot \mathbf{w}_{n}^{T}\right] \mathbf{W}_{n}^{\dagger}\right)-\mathbf{1} \cdot \mathbf{w}_{n-1}^{T}\right] \mathbf{W}_{n-1}^{\dagger} \cdots\right)-\mathbf{1} \cdot \mathbf{w}_{3}^{T}\right] \mathbf{W}_{3}^{\dagger}\right),
\end{aligned}
$$

The process is iterated until the weights of the $n$ th-layer is obtained:

$$
\boldsymbol{W}_{n}=\left[\mathbf{1}, f_{n-1}\left(\cdots f_{2}\left(\left[\mathbf{1}, f_{1}\left(\mathbf{X} \boldsymbol{W}_{1}\right)\right] \boldsymbol{W}_{2}\right) \cdots \boldsymbol{W}_{n-1}\right)\right]^{\dagger} f_{n}^{-1}(\mathbf{Y}) .
$$

Based on the above derivation, the full weights for each layer (i.e., $\boldsymbol{W}_{k}, k=$ $1, \cdots, n)$ can be obtained in an analytic form when the weights without considering the bias component (i.e., $\mathrm{W}_{k}, k=2, \cdots, n$ ) is known. Here, we propose to use a random initialization of $\mathrm{W}_{k}, k=2, \cdots, n$ for solving $\boldsymbol{W}_{k}, k=1, \cdots, n$ in network learning. We shall call this learning network KARnet for convenience.

\section{Synthetic Data}

In this section, we observe the behavior of the proposed network learning on three synthetic data sets with known properties. The first set of data represents the regression problem whereas the second and third data sets are well-known benchmarks for classification. For all the three problems and including the following experiments, our choice of the activation function $f$ and its inverse for each layer are respectively the modified softplus function (i.e., $\left.f(x)=\log \left(0.8+e^{x}\right)\right)$ and its inverse given by $f^{-1}(x)=\log \left(e^{x}-0.8\right)$.

\subsection{Single Dimensional Regression Problem}

The first set of synthetic data has been generated using $y=\sin (2 x) /(2 x)$ based on $x \in\{1,2,3,4,5,6,7,8\}$ for training. To simulate noisy outputs, a $20 \%$ of variation from the original $y$ values has been incorporated where 10 trials of the noisy 
measurements are included for training as well. A two-layer network is adopted to learn the data. Fig. 1(a) shows the learning results for all the eight training data points when a two-layer network uses six hidden nodes (i.e., a 6-1 structure). This is an over-determined system since there are more data samples than the effective number of parameters. Fig. 1(b) shows the results when eight hidden nodes are used for a two-layer network (i.e., a 8-1 structure). This is an under-determined system as there are less data samples than the number of effective parameters (total $8+1$ including the bias's weight). Here, we note that for the two-layer network, the system size is determined by the dimension of $\left[\mathbf{1}, f_{1}\left(\mathbf{X} \boldsymbol{W}_{1}\right)\right]$ and its rank. 1

Next, a five-layer network is used to learn the same set of 1D data. Fig. 2(a) shows the results for the network of 1-1-1-6-1 structure. This constitutes an overdetermined case where there are less effective parameters than data samples). Fig. 2(b) shows the results for the network of 1-1-1-8-1 structure which is underdetermined. Here, the system structure of interest is given by

$$
\left[\mathbf{1}, f_{4}\left(\left[\cdots f_{2}\left(\left[\mathbf{1}, f_{1}\left(\mathbf{X} \boldsymbol{W}_{1}\right)\right] \boldsymbol{W}_{2}\right) \cdots\right)\right] \boldsymbol{W}_{4}\right] .
$$

In summary, the network is seen to find its fit through all data points including those noisy ones for the under-determined case in both networks. However, for the over-determined case, the network does not fit every data points due to the insufficient number of parameters for modelling all data points. This example clearly explains the fitting behavior of multilayer network learning.

\footnotetext{
${ }^{1}$ According to (9)-(11), a Moore-Penrose inverse operation is taken over the matrices (i.e., $\left.\mathbf{X},\left[\mathbf{1}, f_{1}\left(\mathbf{X} \mathbf{W}_{1}\right)\right], \cdots,\left[\mathbf{1}, f_{n-1}\left(\cdots f_{2}\left(\left[\mathbf{1}, f_{1}\left(\mathbf{X} \mathbf{W}_{1}\right)\right] \mathbf{W}_{2}\right) \cdots \mathbf{W}_{n-1}\right)\right]\right)$ to relate among the weight solutions. For the 2-layer network, the matrix $\left[\mathbf{1}, f_{1}\left(\mathbf{X} \mathbf{W}_{1}\right)\right]$ which is nearest to the output is of size $m \times\left(h_{1}+1\right)$. Here, the minimum size of the hidden nodes required for this matrix to be invertible is $\left(h_{1}+1\right)=m$. This in turn gives rise to an output weight of size $\left(h_{1}+1\right) \times q=m \times 1$ for $q=1$. Hence, the effective number of parameters (or adjustable parameters) needed for data representation is hinged upon the number of output weights which corresponds to the sample size and the output dimension (i.e., $m \times q$ ).
} 


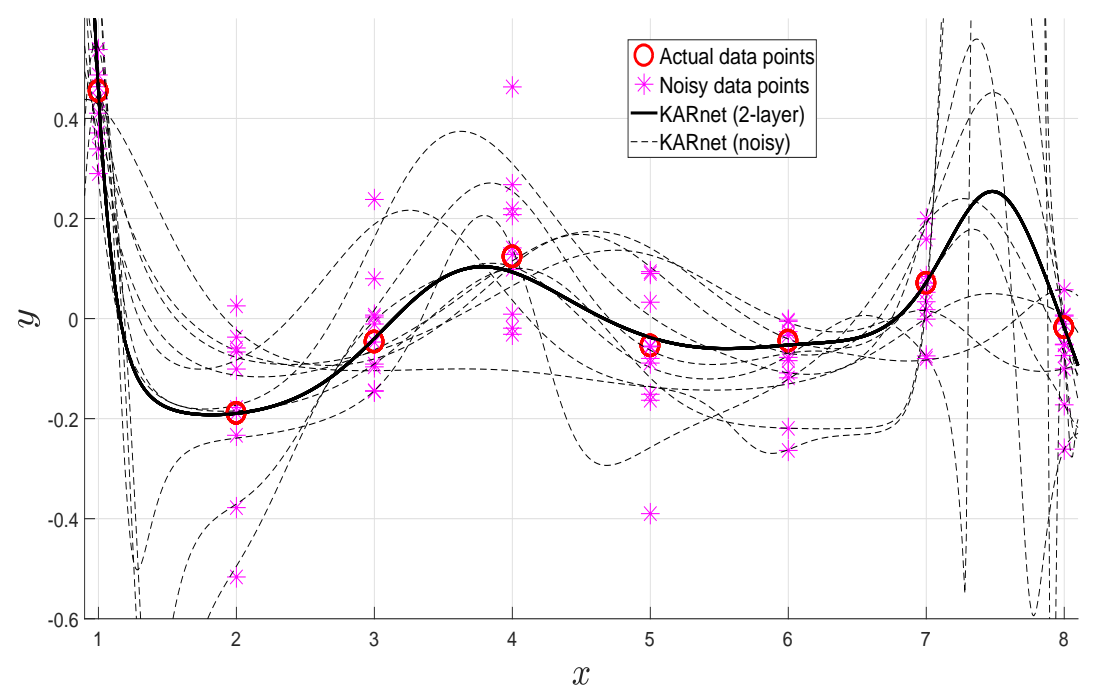

(a)

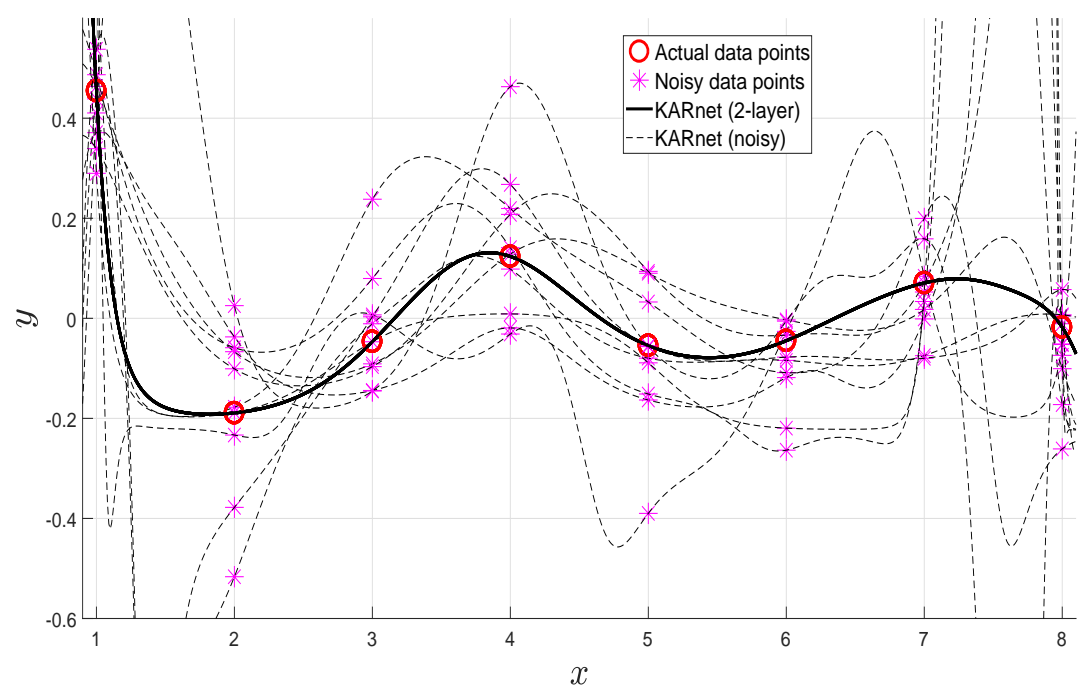

(b)

Figure 1: Decision outputs of a two-layer feedforward network (KARnet with $f=$ softplus) trained by the proposed method. The top and bottom panels demonstrate respectively an under-fitting case and an over-fitting case. 


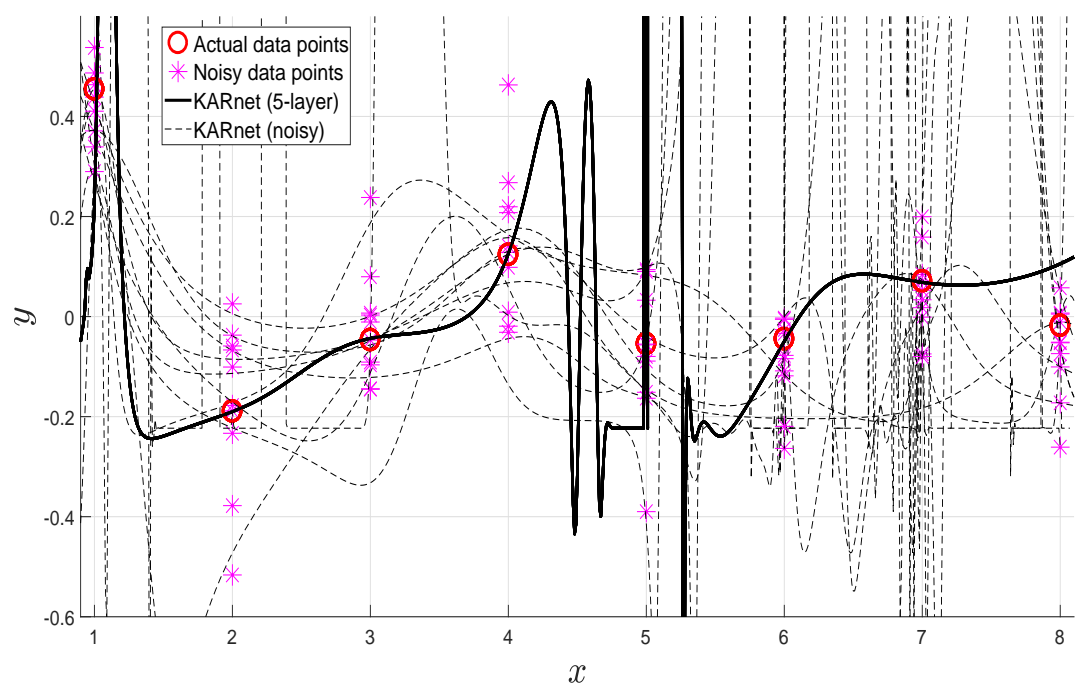

(a)

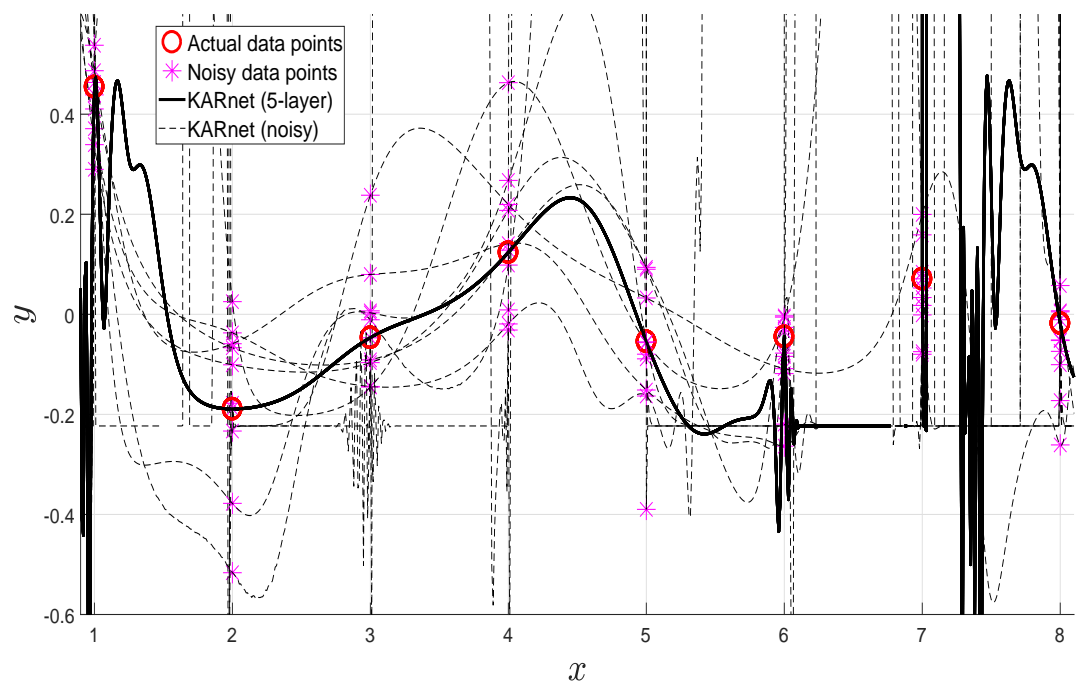

(b)

Figure 2: Decision outputs of a five-layer feedforward network (KARnet with $f=$ softplus) trained by the proposed method. The top and bottom panels demonstrate respectively an under-fitting case and an over-fitting case. 


\subsection{The XOR Problem}

The next example is the well-known XOR problem which consists of four data points with one of the data points being perturbed by a small value to facilitate numerical stability in learning (i.e., the input data points are $\{(0,0),(1,1),(1,0),(0.001,1.001)\}$ which are associated with labels $\{0,0,1,1\}$ respectively). A two-layer KARnet with two hidden nodes is adopted to learn the data. For comparison, the feedforwardnet of the Matlab toolbox is adopted with a similar architecture (adopting a two-layer structure with softplus activation) for learning the same set of data using the default training method trainlm. Fig. 3(a) and (b) show respectively the learned decision surfaces for KARnet and feedforwardnet. These results show the capability of KARnet to fit the nonlinear surface and the premature stopping of feedforwardnet for this data set.

Next, we compare the two networks using a five-layer architecture with each layer having two hidden nodes for both networks. Fig. 4(a) and (b) show respectively the decision surfaces for KARnet and feedforwardnet. These results show the fitting capability of KARnet despite the larger number of adjustable parameters and again, the premature stopping of feedforwardnet for this data set.

\subsection{The Three-Spiral Problem}

In this example, a total of 1500 randomly perturbed data points which form a 3spiral distribution have been used as the training data. Among these data, each of the spiral arm consists of 500 data points (which are shown as red, green and blue circles in Fig. 5). A two-layer KARnet with 100 hidden nodes has been adopted for learning these data points with respective labels using an indicator matrix. Since there are three classes, the number of output nodes is 3 . The learned decision regions (which are shown in light red, green and blue tones) as shown in Fig. 5 show the mapping capability of KARnet for the three-category problem. 


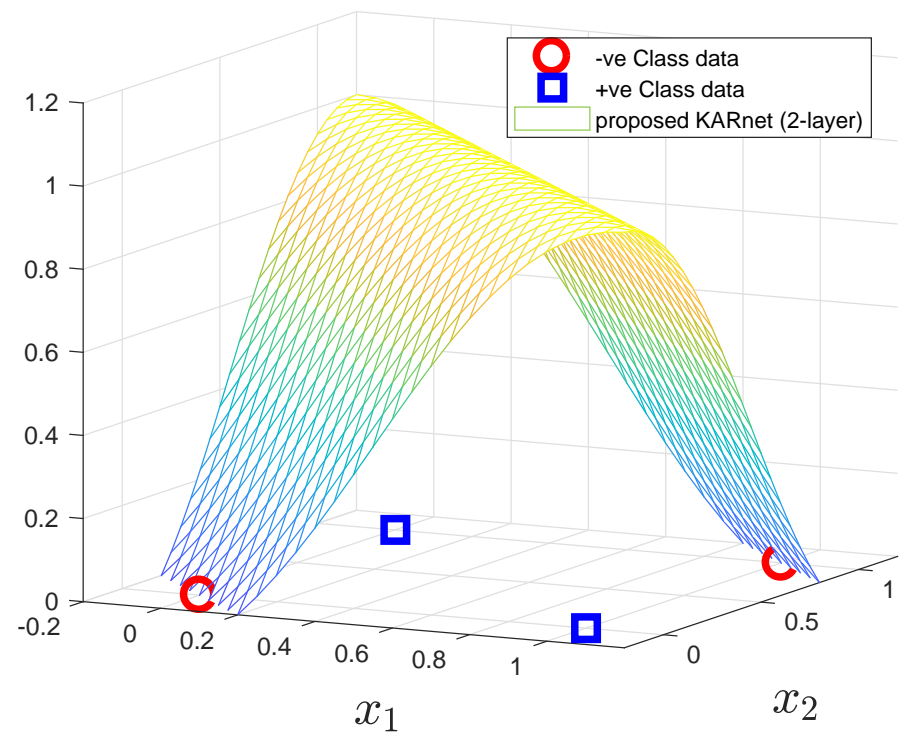

(a)

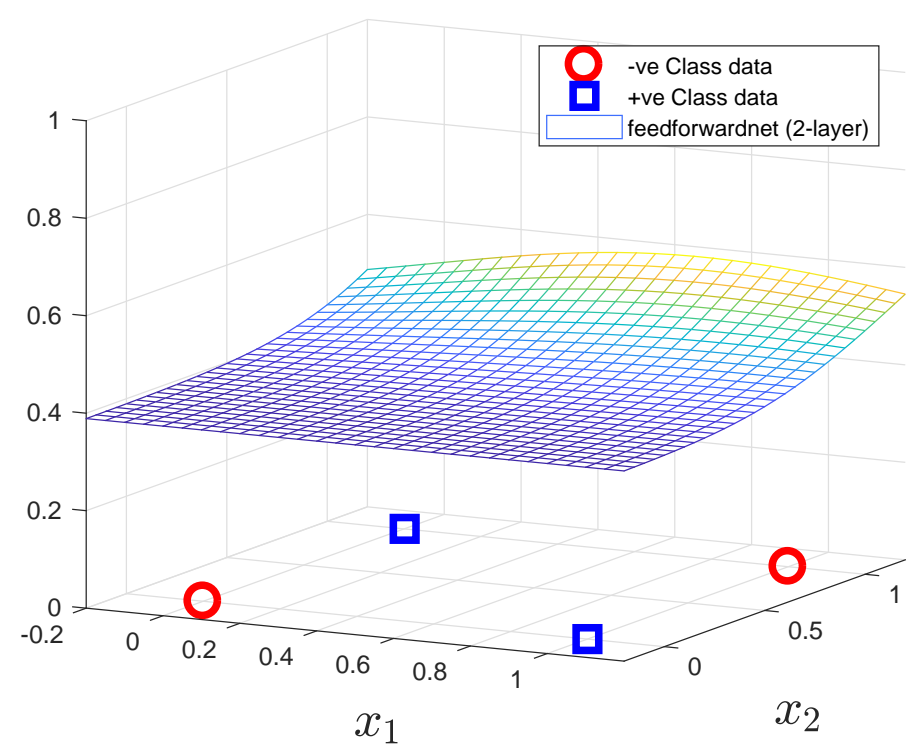

(b)

Figure 3: Decision surfaces of two-layer feedforward networks (the proposed KARnet with $f=$ softplus, and feedforwardnet with $f=$ softplus trained by trainlm). 


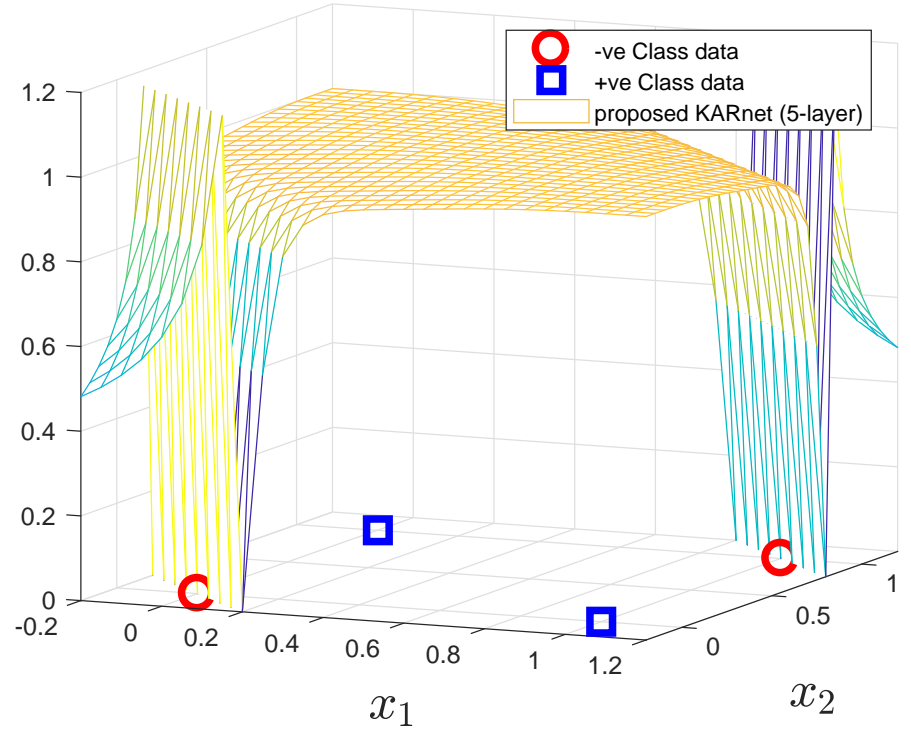

(a)

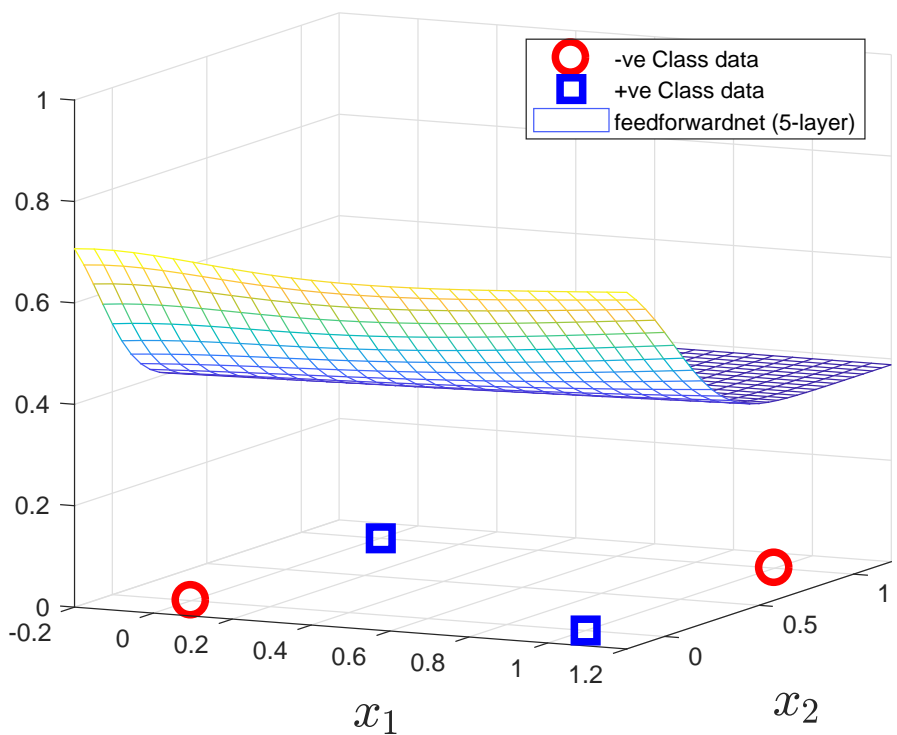

(b)

Figure 4: Decision surfaces of five-layer feedforward networks (the proposed KARnet with $f=$ softplus, and feedforwardnet with $f=$ softplus trained by trainlm). 


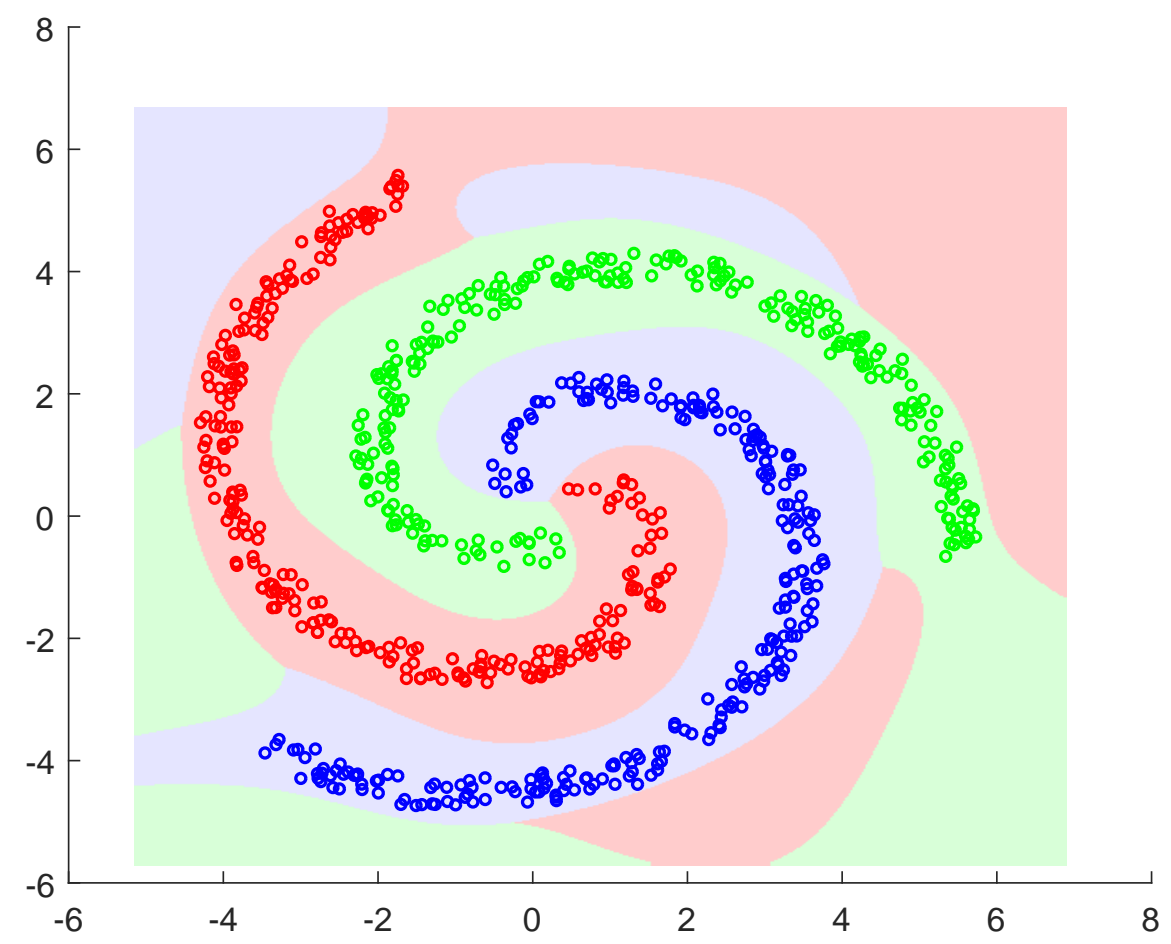

Figure 5: Decision regions of a three-layer feedforward network (proposed KARnet with $f=$ softplus) trained by the proposed method. 


\section{Experiments on Real-World Data}

\subsection{Nursery Data Set}

The goal in this database [21, 22] was to rank applications for nursery schools based upon attributes such as occupation of parents and child's nursery, family structure and financial standing, as well as the social and health picture of the family. The eight input features for the 12960 instances are namely, 'parents' with attributes usual, pretentious, great_pret; 'has_nurs' with attributes proper, less_proper, improper, critical, very_crit; 'form' with attributes complete, completed, incomplete, foster; 'children' with attributes 1, 2, 3, more; 'housing' with attributes convenient, less_conv, critical; 'finance' with attributes convenient, inconv; 'social' with attributes non-prob, slightly_prob, problematic; 'health' with attributes recommended, priority, and not_recom. These input attributes are converted into discrete numbers and normalized to the range $(0,1]$. The output decisions include 'not_recom' with 4320 instances, 'recommend' with 2 instances, 'very_recom' with 328 instances, 'priority' with 4266 instances and 'spec_prior' with 4044 instances. Since the category 'recommend' has not enough instances for partitioning in 10fold cross-validation, it is merged into the 'very_recom' category. We thus have 4 decision categories for classification.

For KARnet's hidden parameter tuning, an inner 10-fold cross-validation loop using only the training set was adopted to determine the hidden node size among $h \in\{1,2,3,5,10,20,30,50,80,100,200,500\}$. For 3-layer and 4-layer networks, the network structures of $2 h-h-q$ and $4 h-2 h-h-q$ are adopted respectively ( $q$ is the output dimension). The chosen hidden node size is then applied for 10 runs of test evaluation using the outer cross-validation loop. The results for 2-layer, 3-layer, and 4-layer networks are respectively $92.39 \%$ at $h=100,92.64 \%$ at $h=80$, and $92.73 \%$ at $h=200$. These results are comparable with $98.89 \%$ for the feedforwardnet ( $h=100,2$-layer) and $91.69 \%$ for the TERRM method [23].

\subsection{Letter Recognition}

The data set comes with 20,000 samples, each with 16 feature attributes. The goal is to recognize the 26 capital letters in the English alphabet based on a large number of black-and-white rectangular pixel displays. The character images consist of 20 different fonts where each letter within these 20 fonts was randomly distorted to produce a large pool of unique stimuli [24, 22]. Each stimulus was converted into 16 primitive numerical attributes such as the statistical moments and the edge counts. These attributes were then scaled to fit into a range of integer values from 0 to 15 .

Similar to the above evaluation setting, 10 trials of 10 -fold stratified crossvalidation have been performed for classifying the 26 categories. The results for 2-layer, 3-layer, and 4-layer KARnets are respectively $88.99 \%, 94.32 \%$, and $94.12 \%$, all at $h=500$. The feedforwardnet (2-layer) encounters "out of memory" for the current computing platform (Intel i7-6500U CPU at $2.59 \mathrm{GHz}$ with $8 \mathrm{G}$ of RAM).

\subsection{Optical Recognition of Handwritten Digits}

This data set was collected based on a total of 43 people, wherein 30 contributed to the training set and different 13 to the test set [25, 22]. The original $32 \times 32$ 
bitmaps were divided into non-overlapping blocks of $4 \times 4$ where the number of on pixels were counted within each block. This generated an input matrix of $8 \times 8$ where each element was an integer in the range [0, 16]. The dimensionality (64) is thus reduced (from $32 \times 32$ ) and the resulted image is invariant to minor distortions. The total number of samples collected for training and testing are respectively 3823 and 1797. In our experiment, these two sets (training and test sets) of data are combined for the running of 10 trials of 10 -fold cross-validation tests. Fig. 6 shows some samples of the image data taken from the training set and the testing set,
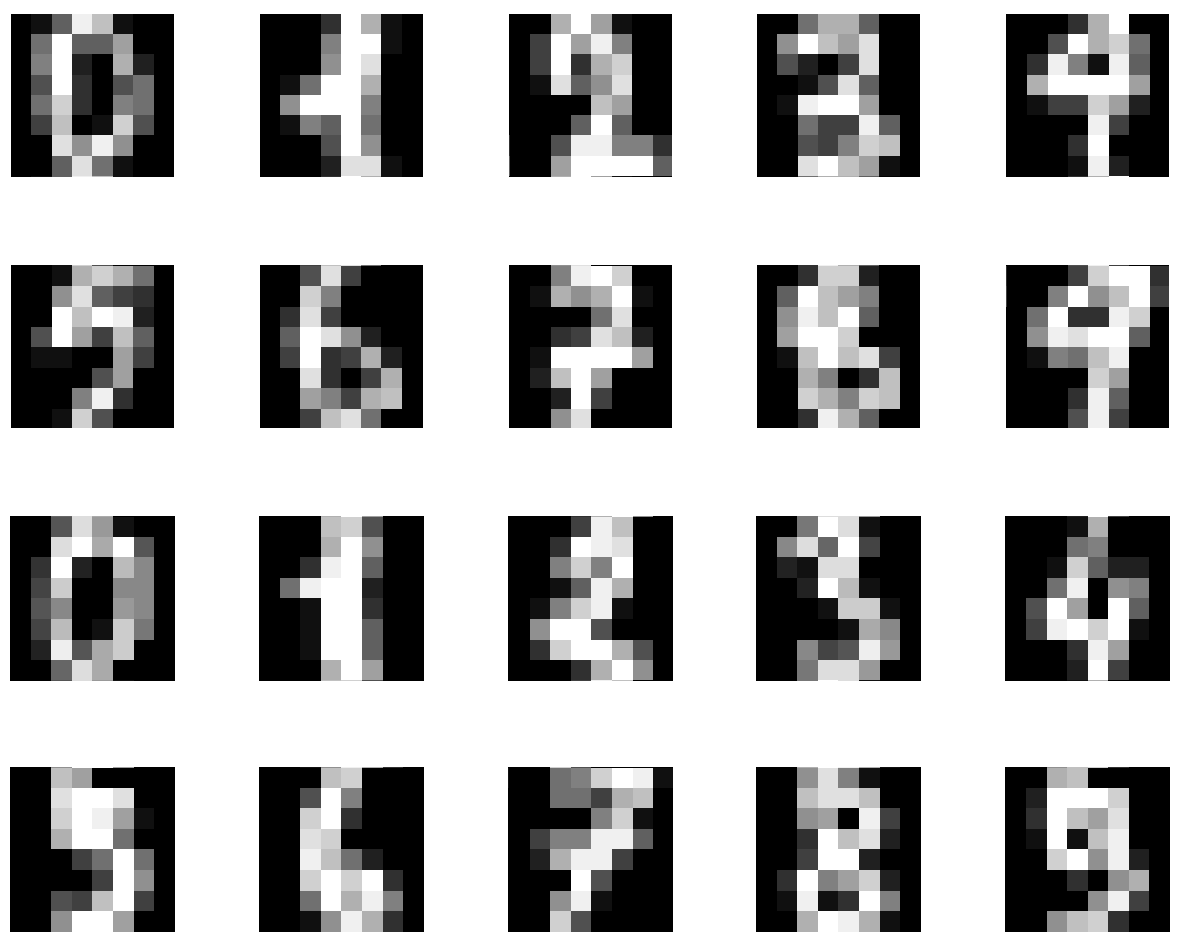

Figure 6: Handwritten digits: samples in the upper two panels are taken from the training set and samples in the bottom two panels are taken from the test set.

The results for 2-layer, 3-layer, and 4-layer networks are respectively $97.25 \%$ at $h=500,97.17 \%$ at $h=200$, and $96.96 \%$ at $h=100$. These results are comparable with the $96.81 \%$ for the TERRM method [23]. The feedforwardnet (2-layer) encounters "out of memory" for the current computing platform.

\subsection{Comparison with State-of-the-arts}

The results of KARnet are compared with several state-of-the-art methods namely, the Reduced Multivariate Polynomial method (RM, [26]), the Total Error Rate method adopting RM (TERRM, 23]), the Support Vector Machines adopting Polynomial (SVM-Poly, [27]) and Radial Basis Function (SVM-Rbf, [27]) kernels, and the feedforwardnet (2-layer) from the Matlab toolbox [28, all running under the similar 10 trials of 10 -fold cross-validation protocol. Table 1 shows that the proposed KARnet has comparable prediction accuracy with state-of-the-art methods. While the SVMs had been tuned by adjusting the kernel parameters (such as the order in the polynomial kernel, and the Gaussian width in the radial basis kernel), 
the proposed network had been tuned by adjusting the number of hidden nodes $(h)$ in each layer according to the structures $2 h-h-q$ and $4 h-2 h-h-q$.

Table 1: Comparison of accuracy (\%) with state-of-the-arts

\begin{tabular}{|l|c|c|c|}
\hline Methods & Nursery & Letter & Optdigit \\
\hline RM [26] & 90.93 & 74.14 & 95.32 \\
TERRP [29] & 96.46 & 88.20 & 98.16 \\
TERRM [23] & 91.69 & 78.42 & 96.81 \\
SVM-Poly [27] & 91.61 & 77.22 & 95.52 \\
SVM-Rbf [27] & 98.24 & 97.14 & 99.13 \\
FFnet(2-layer) [28] & 98.89 & OM & OM \\
KARnet(2-layer) & 92.39 & 88.99 & 97.25 \\
KARnet(3-layer) & 92.64 & 94.32 & 97.17 \\
KARnet(4-layer) & 92.73 & 94.12 & 96.96 \\
\hline
\end{tabular}

FFnet : Feedforwardnet from Matlab [28].

OM : Out of memory for the current computing platform.

\section{Conclusion}

In this article, the solution based on the manipulation of the kernel and the range space has been found to be equivalent to that obtained by the least squares error estimation. By exploiting this observation, a learning approach based on the kernel and the range space manipulation has been introduced. The approach solves the system of linear equations directly by exploiting the row and the column spaces without the need for error formulation and gradient descent. The adoption of the learning approach to deep networks learning validated its feasibility. The learning results of synthetic and real-world data provided not only the numerical evidence but also the insights regarding the fitting mechanism. This opens up the vast possibilities along the research direction.

\section{Acknowledgment}

This research was supported by Basic Science Research Program through the National Research Foundation of Korea (NRF) funded by the Ministry of Education, Science and Technology (Grant number: NRF-2015R1D1A1A09061316). 


\section{References}

[1] H. J. Kelley, "Gradient theory of optimal flight paths," Ars Journal, vol. 30, no. 10, pp. 947-954, 1960.

[2] P. J. Werbos, "Beyond regression: New tools for prediction and analysis in the behavioral sciences," Ph.D. dissertation, Beyond Regression: New Tools for Prediction and Analysis in the Behavioral Sciences, 1974.

[3] — , "Backpropagation : Past and future," in ICNN proceedings (IEEE), 1988.

[4] - "Backpropagation through time: what it does and how to do it," Neural Networks, vol. 78, no. 10, pp. 1550-1560, 1990.

[5] S. O. Haykin, Neural Networks and Learning Machines. New York: Prentice Hall, 2009.

[6] K.-I. Funahashi, "On the approximate realization of continuous mappings by neural networks," Neural Networks, vol. 2, no. 3, pp. 183-192, 1989.

[7] K. Hornik, M. Stinchcombe, and H. White, "Multi-layer feedforward networks are universal approximators," Neural Networks, vol. 2, no. 5, pp. 359-366, 1989 .

[8] G. Cybenko, "Approximations by superpositions of a sigmoidal function," Math. Cont. Signal \& Systems, vol. 2, pp. 303-314, 1989.

[9] R. Hecht-Nielsen, "Kolmogorov's mapping neural network existence theorem," in Proceedings of IEEE First International Conference on Neural Networks (ICNN), vol. III, 1987, pp. 11-14.

[10] R. Battiti, "First and second order methods for learning: Between steepest descent and newtons method," Neural Computation, vol. 4, no. 2, pp. 141166, 1992.

[11] P. Patrick van der Smagt, "Minimisation methods for training feedforward neural networks," Neural Networks, vol. 7, no. 1, pp. 1-11, 1994.

[12] E. Barnard, "Optimization for training neural nets," IEEE Trans. on Neural Networks, vol. 3, no. 2, pp. 232-240, 1992.

[13] I. Goodfellow, Y. Bengio, and A. Courville, Deep Learning. MIT Press, 2016, http://www.deeplearningbook.org.

[14] W. R. Madych, "Solutions of underdetermined systems of linear equations," in Lecture Notes - Monograph Series, Spatial Statistics and Imaging, 1991, vol. 20, pp. 227-238, institute of Mathematical Statistics.

[15] S. Boyd and L. Vandenberghe, Convex Optimization. Cambridge: Cambridge University Press, 2004.

[16] A. Albert, Regression and the Moore-Penrose Pseudoinverse. New York: Academic Press, Inc., 1972, vol. 94. 
[17] Adi Ben-Israel and Thomas N.E. Greville, Generalized Inverses: Theory and Applications, 2nd ed. New York: Springer-Verlag, 2003.

[18] S. L. Campbell and C. D. Meyer, Generalized Inverses of Linear Transformations, (SIAM edition of the work published by Dover Publications, Inc., 1991) ed. Philadelphia, USA: Society for Industrial and Applied Mathematics, 2009.

[19] G. Strang, Introduction to Linear Algebra, 5th ed. Wellesley: WellesleyCambridge Press, 2016.

[20] R. O. Duda, P. E. Hart, and D. G. Stork, Pattern Classification, 2nd ed. New York: John Wiley \& Sons, Inc, 2001.

[21] M. Olave, V. Rajkovic, and M. Bohanec, "An application for admission in public school systems," in Expert Systems in Public Administration, I. Th. M. Snellen, W. B. H. J. van de Donk, and J.-P. Baquiast, Eds. North Holland: Elsevier Science Publishers, 1989, pp. 145-160.

[22] M. Lichman, "UCI machine learning repository," 2013. [Online]. Available: http://archive.ics.uci.edu/ml

[23] K.-A. Toh and H.-L. Eng, "Between classification-error approximation and weighted least-squares learning," IEEE Trans. Pattern Analysis and Machine Intelligence, vol. 30, no. 4, pp. 658-669, April 2008.

[24] P. W. Frey and D. J. Slate, "Letter recognition using holland-style adaptive classifiers," Machine Learning, vol. 6, no. 2, pp. 161-182, 1991.

[25] C. Kaynak, "Methods of combining multiple classifiers and their applications to handwritten digit recognition," Master's thesis, Institute of Graduate Studies in Science and Engineering, Bogazici University, 1995.

[26] K.-A. Toh, Q.-L. Tran, and D. Srinivasan, "Benchmarking a reduced multivariate polynomial pattern classifier," IEEE Trans. on Pattern Analysis and Machine Intelligence, vol. 26, no. 6, pp. 740-755, 2004.

[27] C.-C. Chang and C.-J. Lin, "LIBSVM: A library for support vector machines," ACM Transactions on Intelligent Systems and Technology, vol. 2, pp. 27:127:27, 2011, software available at http://www.csie.ntu.edu.tw/ cjlin/libsvm.

[28] The MathWorks, "Matlab and simulink," in [http://www.mathworks.com/], 2017.

[29] K.-A. Toh, "Deterministic neural classification," Neural Computation, vol. 20, no. 6 , pp. 1565-1595, June 2008. 